\title{
A New Approach for Real-time Iris Liveness Detection Based on Eye Movement's Features
}

\author{
Pham The Bao \\ Computer Science Department, Information Science Faculty, Sai Gon University, Vietnam \\ ptbao@sgu.edu.vn,ptbao2005@gmail.com
}

\begin{abstract}
This research introduces a new approach for iris liveness detection based on eye movement's features. Given 50 valid eye frames from real-time video stream, this research identify them as the images that were taken whether directly from humans' eyes or not. Our method ensures the selected images for further processing are eye ones without user's cooperation during the sampling process. In addition, it also controls the application timeout by identifying either fake iris or low quality eye images. In my study, this Research used two features to model eye movements: (1) the width/height ratio of the eye outline and (2) the distance from the pupil center to the eye centroid. In order to model the acquired data distribution, this research used a bag of two-component Gaussian Mixing Models (GMMs). The class label of the acquired data depends on the GMMs which it belongs. The average accuracy of my proposed method on my and IriTech, Inc.'s databases was $94.704 \%$ and $100 \%$, respectively. This result indicated that my method can make real-time iris-based systems work automatically with high security.
\end{abstract}

Index Terms: Bagging, correlation template matching, eye movements, eye tracking, Gaussian mixing model, iris liveness detection.

\section{Introduction}

Now days, with the development trend of smart portable devices, not only can this research manage our private information such as banking accounts, but also our business just by a small device, e.g., a tablet, a phablet or a smartphone. As a result, this research need a higher security level technique. Additionally, the traditional security methods such as password, PIN or card technology are not the best selection to protect our information. One of many reasons is that it is not comfortable because users have to remember their password strings or carry cards, keys while they usually own more than one account. Therefore, biometrics authentication with high security level are getting popular and will be a useful replacement for privacy protection [1]. Besides, iris-based security system significantly shows the outstanding advantages: unique, stable, flexible, reliable, and noninvasive [2].

Unfortunately, as other forms of biometrics authentications, there are many ways to attack iris identification system, by means of printed iris image, artificial eyeball, "eye movie" or even a complex counterfeit way using contact lens [3]. According to [4], with the simple tool such as printed iris image, the successful authentication rate can be greater than $50 \%$. As a result, this requires the development of fake iris detection to prevent unauthorized uses.

There are two basic approaches in this research area: hardware-based and software-based [5]. Regarding the first one, its performance and accuracy gave the impressive results in [6], [7], [8], and [9]. However, software-based approach is a promising long-term development based on available technical equipment [5] and easier to apply to portable devices. One of the problems in this research branch is how to establish good features to separate between fake and liveness irises. Recently, the research community has paid more attention in the reflectance of human's pupil when luminous intensity is changed, which is used in [3], [10], [11], [12], [13], and [14]. However, with a stable sampling environment and a nonflash iris camera, the luminous intensity variation converges to zero, that is, pupil's size is not changed during the sampling process. Besides, Chen et al. introduced a method using minutiae points in conjunctival vessels and the

Received: December $4^{\text {th }}, 2019$. Accepted: September $30^{\text {th }}, 2020$

DOI: $10.15676 /$ ijeei.2020.12.4.3 
entropy ratio of iris textures [15]. Because this technique requires good quality and highresolution images, high technology in iris cameras is needed.

Based on image, Javier Galbally [27] used private database to detect iris liveness on image by classify fake or real iris, the highest accuracy is $24.00 \%$ without running time. Adam Czajka [37] presented his method to iris liveness detection by modeling dynamic pupil features, his method spend $200 \mathrm{~ms}$ for an image; after that he survey some methods to classify fake or real iris on image [28]. Rohit Agarwal [29] have given the highest accuracy is $99.1 \%$ for iris liveness detection by enhanced binary hexagonal extrema pattern descriptor of image. Niladri B. Puhan [30], André Anjos [31], Yang Hu [33] suggested some new ideas, but they didn't the result and performance. Federico Pala [34] compared results on GPU and CPU is $5.2 \mathrm{~ms}$ on a single GPU and $14 \mathrm{~ms}$ on the CPU. The average classification error is from $0 \%$ to $17.2 \%$. Ana F.Sequeira [38] combined private and public data to experimented, the associated error is $13.2-43.6$. Gabriela Yukari Kimura used CNN hyper-parameter tuning then the error rates depends parameters from 0 to $49.85 \%$ [36].

Based on video, Kiran B. Raja and Et al [35] applied private database to their method. The average classification error rate is $0 \%$ after 11 frames. Diego Gragnaniello [32] used local descriptors to classify fake or real iris on private database. The running time is from $0.2 \mathrm{~ms}$ to $256 \mathrm{~ms}$ for a frame.

In this paper, this research introduce a new method for iris liveness detection using a new feature: eye movements, which is a basic characteristic of liveness eyes. According to the biological studies about eye movements in [16] and [17], this research blink about 15-20 times and make greater than or equal to 40 saccades per minute. Therefore, by tracking eyelids and pupil, this research detect eye movements via the width/height ratio of the eye outline and the distance from the pupil center to the eye centroid. Our proposed method uses 50 valid eye images from the video stream in order to measure eye movement rate and make decision based on this parameter. Moreover, this research can select the most informative iris image from the captured images using our previous research [18]. Then, this research use a bag of two-component Gaussian Mixing Models (GMMs) to model the acquired features distribution. The class label of the acquired data depends on the GMMs which it belongs to. In my experiment, this research construct a database with the Irishield MK $2120 \mathrm{U}$ iris scanner for training and testing purposes. In addition, this research evaluate my method on the IriTech, Inc.'s database. The average accuracy of my proposed method on my and the IriTech, Inc.'s databases is $94.704 \%$ and $100 \%$, respectively.

\section{Methodology}

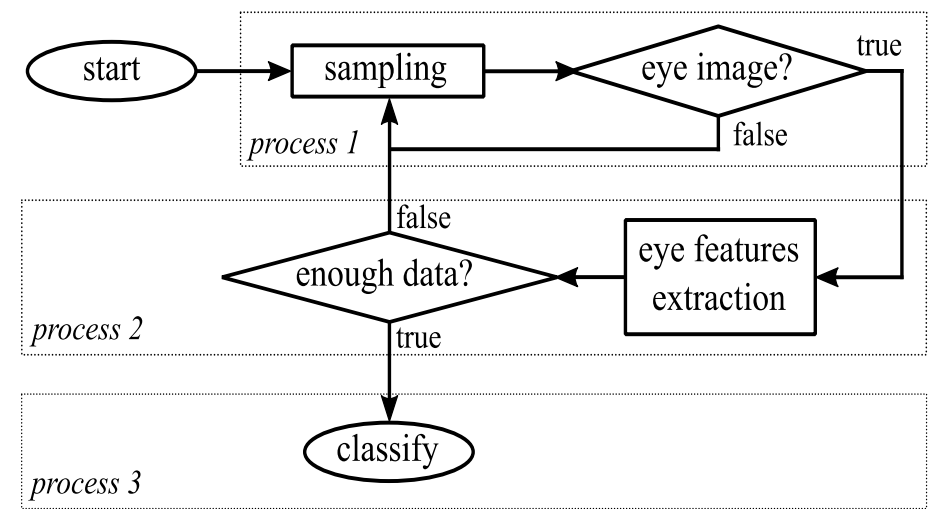

Flowchart 1. Schematic of my proposed method.

Our proposed method is described as Flowchart 1. In order to build a system that works automatically without user's cooperation, in the first process, this research ensure that the 
selected images for further processing are eye ones using correlation template matching method (CTMM). Furthermore, this process controls my application timeout by identifying fake iris images whose the quality is not good enough. Given the valid eye images from the process 1 , this research introduce a technique to detect eye movements via the width/height ratio of the eye outline and the distance from the pupil center to the eye centroid. Finally, this research use GMMs to classify the acquired data.

\section{A. Eye Existence Verification}

In our previous research [18], this research used Haar cascade classifier in order to detect eye in an image since this research dealt with the acquired data from liveness eye only. The advantages of this approach are high accuracy and speed in case the quality of input images are good. On the other hand, the true detection rate of this method is low with poor quality images, which can cause errors in my next processing steps. In this research, the aim is to detect authentication attacks using good as well as poor quality images; hence, this method cannot meet our demand.

In this study, this research use CTMM to locate eye in an image. Given true eye images $T^{1}, T^{2}, \ldots, T^{N} \in \mathrm{N}^{h \times w}$, this research generate a valid eye template using (1),

$$
T_{t}(x, y)=\min \left\{\frac{\sum_{i=1}^{N} T^{i}(x, y)}{N}, 255\right\} .
$$

where $T_{t}(x, y)$ and $T^{i}(x, y)$ are the intensities of the pixel at $(x, y)$ in $T_{t}$ and $T^{i}$, respectively. Similarly, this research apply (1) to create eye template $T_{f}$ in order to reject invalid eye in an image. Figure. 1 shows the valid eye templates this research use in this research. In addition, this research use invalid eye templates in Figure. 2 to reject unaccepted eye in an image.

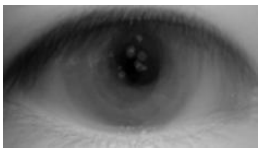

(a) Normal-size right eye $T_{t}^{1}$.

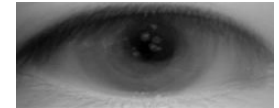

(c) Small-size right eye $T_{t}^{3}$.

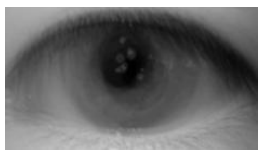

(b) Normal-size left eye $T_{t}^{2}$.

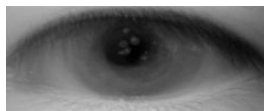

(d) Small-size left eye $T_{t}^{4}$.

Figure 1. Valid eye templates.
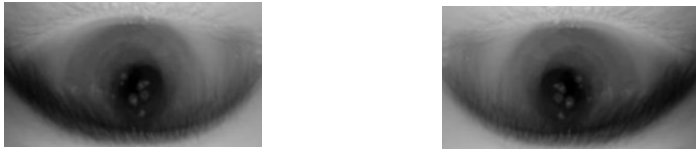

(a) Invalid normal-size right eye $T_{f}^{1}$.(b) Invalid normal-size left eye $T_{f}^{2}$.
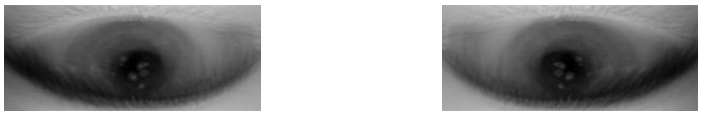

(c) Invalid small-size right eye $T_{f}^{3}$.(d) Invalid small-size left eye $T_{f}^{4}$.

Figure 2. Invalid eye templates. 
Let $I \in \mathrm{N}^{240 \times 320}$ be an input image, this research establish function $h$ in order to detect eye in an image and estimate its location,

$$
\begin{aligned}
h:\left(\mathrm{Z}^{13 \times 23}, \mathrm{Z}^{24 \times 32}\right) & \rightarrow(\mathrm{R}, \mathrm{Z}, \mathrm{Z}) \\
\left(T_{s}^{i}, I\right) & \mapsto\left(c^{s, i}, x_{0}^{s, i}, y_{0}^{s, i}\right), i \in\{1,2,3,4\}, s \in\{t, f\}
\end{aligned}
$$

where $c^{s, i} \in[-1,1]$ is the correlation between $T_{s}^{i}\left(x_{0}^{s, i}, y_{0}^{s, i}\right)$ and $I\left(x_{0}^{s, i}, y_{0}^{s, i}\right)$ which is calculated by (2) [19],

$$
c^{s, i}=\frac{\sum_{x^{\prime}, y^{\prime}} T_{s}^{i}\left(x^{\prime}, y^{\prime}\right) * I\left(x_{0}^{s, i}+x^{\prime}, y_{0}^{s, i}+y^{\prime}\right)}{\sqrt{\sum_{x^{\prime}, y^{\prime}} T_{s}^{i}\left(x^{\prime}, y^{\prime}\right) * \sum_{x^{\prime}, y^{\prime}} I\left(x_{0}^{s, i}+x^{\prime}, y_{0}^{s, i}+y^{\prime}\right)^{2}}} .
$$

Since our template database consists of invalid eye images, this research construct (3) and (4) to Figureure out whether the detected object is valid or not for further processing

$$
i_{0}=\left\{\begin{array}{ll}
i & \text { if } \underset{s}{\operatorname{argmax}}\left\{c^{s, i}\right\}=t \\
i+4 & \text { otherwise }
\end{array}, i=1,2,3,4 .\right.
$$

and

$$
I \text { is a(n) }\left\{\begin{array}{ll}
\text { valid eye image } & \text { if } i_{0} \leq 4 \text { and } t_{c} \leq c^{t, i_{0}} \\
\text { invalid eye image } & \text { otherwise }
\end{array} .\right.
$$

where the correlation threshold $t_{c}=0.939$. Note that $T_{s}^{i}$ and $I$ are $\frac{1}{10}$ times the size of the original $T_{s}^{i}$ and $I$, respectively. This research resize these images before feeding them into $h$ to decrease computational complexity. Figure. 3 presents the results of CTMM in eye detection with regard to liveness and printed eye images.

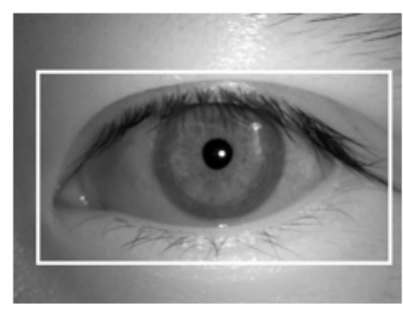

(a) Liveness eye image.

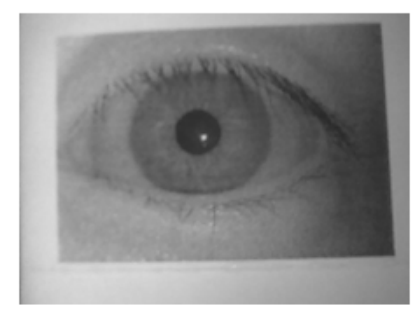

(b) Printed eye image.

Figure 3. Eye detection using CTMM with regard to liveness and printed eye images.

\section{B. Eye Tracking}

Generally, pixel intensities of the pupil are the smallest in an eye image. Thus, performing pupil tracking and using the computed parameters in eyelids tracking is more common. However, without any assumptions, this approach will fail in these cases: (1) image with closed eye or (2) pupil image influenced by hair or eyelashes. From this point of view, in this research, our method performs eyelids tracking first.

\section{1). Upper and Lower Eyelid Limits Estimation}

Suppose $y_{t}, y_{b}, x_{l}$, and $x_{r}$ are the upper, lower, left, and right limits of the eye calculated by CTMM, respectively. In this step, this research recalculate $y_{t}$ and $y_{b}$ to make them approach the better outcomes $y_{t}^{\prime}$ and $y_{b}^{\prime}$. In order to enhance horizontal lines in an eye image, including upper and lower eyelids boundaries, this research apply rank filter [20] with $M=19, k=2$ and 
Canny algorithm with the low threshold $t_{l}=50$ and the high threshold $t_{h}=100$ [21] (Figure. 4). Mathematically, an eye image is a matrix (5)

$$
I=\left[\begin{array}{ccc}
i_{1,1} & \cdots & i_{1,320} \\
\vdots & \ddots & \vdots \\
i_{240,1} & \cdots & i_{240,320}
\end{array}\right] .
$$

with $i_{k, h} \in\{0,1, \ldots, 255\}, k=\overline{1,240}$ and $h=\overline{1,320}$. This research define function $\phi$ as (6).

$$
\phi(i)=\left\{\begin{array}{ll}
1 & \text { if } i=0 \\
0 & \text { otherwise }
\end{array}, \forall i=\overline{0,255} .\right.
$$

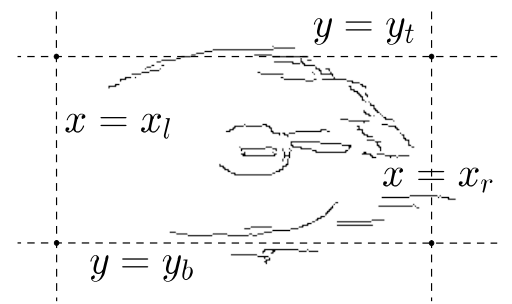

Figure 4. Eye outline.

In addition, this research construct function $V(x, y)$ in (7) to estimate the number of neighbors of the pixel $(x, y)$ who's the intensity are equal to zero,

$$
V(x, y)=\sum_{k=x_{l}-\frac{\left(x_{r}-x_{l}\right)}{3}}^{x_{r}+\frac{\left(x_{r}-x_{l}\right)}{3}} \sum_{h=y-2}^{y+1} \phi\left(i_{h, k}\right) .
$$

where $i_{h, k}$ is the intensity of the pixel $(h, k)$. Let compute $\mathrm{X}$ by (8). The better outcome $y_{t}^{\prime}$ of $y_{t}$ can be calculated by (9) and this research recalculate $y_{b}^{\prime}$ of $y_{b}$ using (9).

$$
\begin{aligned}
& X=x_{l}+\frac{x_{r}-x_{l}}{2} . \\
& y_{t}^{\prime}=\underset{y}{\operatorname{argmax}} V(X, y), y=y_{t}-15, y_{t}+\frac{\left(y_{b}-y_{t}\right)}{5} .
\end{aligned}
$$

\section{2). Pupil Existence Verification}

According to Kobayashi et al. [22], the human width/height ratio of the eye outline is less than or equal to $t_{p} \approx 2.8$. However, based on my experiments, this research set $t_{p}$ to 2.500 since this research deal with opened eye images only. Let $I$ be an eye image with corresponding parameters $y_{t}, y_{b}, x_{l}$, and $x_{r} . I$ is a valid eye image, i.e., image with opened eye if and only if $I$ 's parameters satisfy (10).

$$
\frac{x_{r}-x_{l}}{y_{b}-y_{t}} \leq t_{p} .
$$

\section{3). Pupil Estimation}

In our previous research [18], in order to denoise a valid eye image, this research use median filter with kernel's size $25 \times 25$ (Figure. 5). Figure. 6a demonstrates the filtered image after 
binarizing with threshold $t_{b}=240$. In addition, this research introduced rhombus method to remove the remaining noise(s) in a pupil image. this research defined dataset $\mathrm{R}$ as (11).

$$
\mathrm{R}=\left\{I(x, y): x_{l} \leq x \leq r_{r}, y_{t} \leq y \leq y_{b}\right\} .
$$

Let $D$ be a rhombus inscribed in $R$ (Figure. 6b). This research construct the dataset $\bar{D}=R \backslash D$ to remove noise(s) outside the rhombus $D$. This method denoises eye image without affecting pupil area because the shapes of rhombus $D$ and the eyelids are similar. This research define $D_{1}$ and $D_{2}$ as two of $D$ 's vertices (Figure. $6 \mathrm{c}$ ) and their coordinates are calculated by (12) and (13).

$$
\begin{aligned}
& \left(x_{\mathrm{D}_{1}}, y_{\mathrm{D}_{1}}\right)=\left(x_{l}, y_{t}+\frac{y_{b}-y_{t}}{2}\right) . \\
& \left(x_{\mathrm{D}_{2}}, y_{\mathrm{D}_{2}}\right)=\left(x_{l}+\frac{x_{r}-x_{l}}{2}, y_{b}\right) .
\end{aligned}
$$

Then $p_{1}=\frac{y_{\mathrm{D}_{1}}-y_{\mathrm{D}_{2}}}{x_{\mathrm{D}_{1}}-x_{\mathrm{D}_{2}}}$ and $p_{2}=y_{\mathrm{D}_{1}}-p_{1} x_{\mathrm{D}_{1}}$ are the slope and the other parameter of the line equation $y=p_{1} x+p_{2}$ respectively given $D_{1}$ and $D_{2}$. Recall $\bar{D}=R \backslash D$ as the set of data points outside the rhombus $\mathrm{D}$. This research constructed this set using (14).

$$
\overline{\mathrm{D}}=\bigcup_{i=1}^{4} \overline{\mathrm{D}_{i}} \text {. }
$$

$$
\begin{aligned}
& \text { Where } \\
& \begin{aligned}
\overline{\mathrm{D}_{1}} & =\{(i, j)\}, \\
\overline{\mathrm{D}_{2}} & =\left\{\left(i, x_{r}-\left(j-x_{l}\right)\right)\right\}, \\
\overline{\mathrm{D}_{3}} & =\left\{\left(y_{t}+y_{b}-i, j\right)\right\}, \\
\overline{\mathrm{D}_{4}} & =\left\{\left(y_{t}+y_{b}-i, x_{r}-\left(j-x_{l}\right)\right)\right\}, \\
i & =\overline{y_{\mathrm{D}_{1}}, y_{b}}, j=\overline{x_{l}, k} \text { and } k=\left(i-p_{2}\right) / p_{1} .
\end{aligned}
\end{aligned}
$$

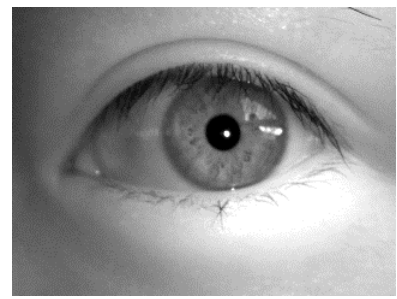

(a) Original image.

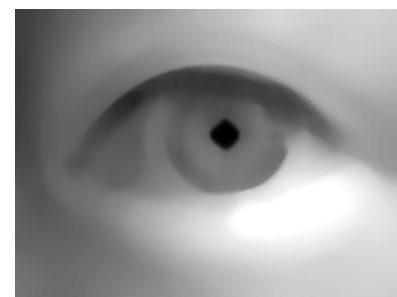

(b) Filtered image.

Figure 5. Median filter. 


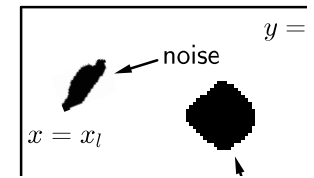

(a) Eye image binarization.

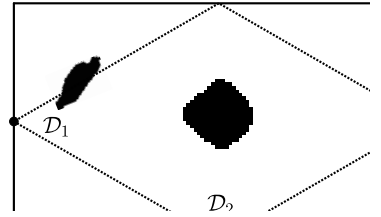

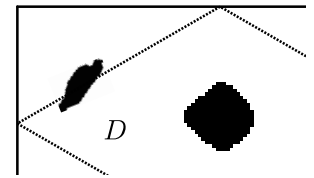

(b) Rhombus method.

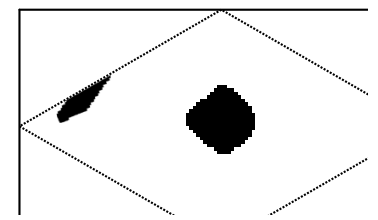

(c) Rhombus method (cont.). (d) Eye image after denoising.

Figure 6. Eye image denoising with rhombus method.

By setting all the pixels $p \in \overline{\mathrm{D}}$ to the background intensity, this research eliminate all noises outside domain $\mathrm{D}$ as Figure. 6d. Finally, let $C=\left\{C_{i}\right\}_{i=1}^{n}$ be a set of $n$ connected zero-value components in the processed image, the pupil region $\mathrm{P}$ is selected by (16).

$$
\mathrm{P}=\underset{C_{i} \in C}{\operatorname{argmin}}\left\{\left\|p_{e}-p_{C_{i}}\right\|\right\} \text {. }
$$

where $p_{e}=\left(X, \frac{x_{r}-x_{l}}{2}\right)$ and $p_{C_{i}}$ is the $C_{i}$ centroid. Then this research calculate the radius $r$ of the pupil using (17). Thus the coordinates $\left(x_{0}, y_{0}\right)$ of the pupil centroid is computed by (18).

$$
r=\frac{1}{2} \max \left\{w_{p}, h_{p}\right\} .
$$

where

$$
\begin{aligned}
& p_{l}=\min \{x:(x, y) \in \mathrm{P}\}, p_{r}=\max \{x:(x, y) \in \mathrm{P}\}, \\
& p_{t}=\min \{y:(x, y) \in \mathrm{P}\}, p_{b}=\max \{y:(x, y) \in \mathrm{P}\}, \\
& w_{p}=p_{r}-p_{l}, \\
& h_{p}=p_{b}-p_{t} . \\
& \left(x_{0}, y_{0}\right)=\left(p_{l}+\frac{p_{r}-p_{l}}{2}, p_{t}+\frac{p_{b}-p_{t}}{2}\right) .
\end{aligned}
$$

\section{4). Pupil Recalculation}

In the Pupil Estimation section, this research introduced a technique to approximate pupil's size and location. Because of using median filter in image denoising, some pixels in the actual pupil border are lost. Hence, it is worthwhile to improve the estimated pupil radius in (17). In this section, this research propose a method to recalculate this parameter using rank filter and Canny algorithm.

Given $\left(x_{0}, y_{0}\right)$ in (18) and $r$ in (17), this research discover four actual pupil limits $T\left(x_{T}, y_{T}\right)$, $B\left(x_{B}, y_{B}\right), L\left(x_{L}, y_{L}\right)$, and $R\left(x_{R}, y_{R}\right)$ as shown in Figure. 7 . In order to locate $T$ and $B$, this 
research apply horizontal rank filter and Canny algorithm (see Figure. 8) to the original eye image. Let $T_{0}\left(x_{T}^{0}, y_{T}^{0}\right)$ and $B_{0}\left(x_{B}^{0}, y_{B}^{0}\right)$ be the initial points of $T$ and $B$ respectively, then

$$
\begin{aligned}
& x_{T}^{0}=x_{0}, y_{T}^{0}=y_{0}-r, \\
& x_{B}^{0}=x_{0}, \quad y_{B}^{0}=y_{0}+r .
\end{aligned}
$$

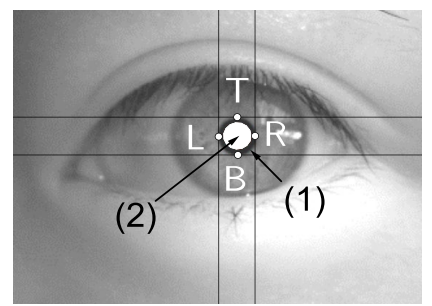

Figure 7. The actual pupil limits: $T, B, L$, and $R$. Note that object (1) and object (2) are the actual and the estimated pupils, respectively.

Assume $I_{h}=\left\{I_{h}(i, j)\right\}$ to be the horizontal-rank filtered image in Figure. 8, where $I_{h}(i, j)$ is the intensity of the pixel $(i, j)$. This research define the set of possible points $C_{T}$ which is related to $T$ by (20).

$$
\begin{aligned}
& C_{T}^{1}=\left\{y_{T}^{\prime}: I_{h}\left(x_{T}^{0}, y_{T}^{\prime}\right)=0, I_{h}\left(x_{T}^{0}, y_{Y}^{\prime}-1\right)=255\right\}, \\
& C_{T}^{2}=\left\{y_{T}^{0}, y_{T}^{0}-1, \ldots, y_{T}^{0}-e_{T}\right\}, \\
& C_{T}=C_{T}^{1} \cap C_{T}^{2} .
\end{aligned}
$$

where $e_{T}=7.000$ is the maximum different value between $y_{T}^{0}$ and $y_{T}$. Then, this research calculate $y_{T}$ using (21) and let $C_{B}, C_{L}$, and $C_{R}$ be the candidate sets related to $B, L$, and $R$ points respectively (22)

$$
\begin{aligned}
& y_{T}=\max \left\{C_{T}\right\} . \\
& C_{B}^{1}=\left\{y_{B}^{\prime}: I_{h}\left(x_{B}^{0}, y_{B}^{\prime}\right)=0, I_{h}\left(x_{B}^{0}, y_{B}^{\prime}+1\right)=255\right\}, \\
& C_{B}^{2}=\left\{y_{B}^{0}, y_{B}^{0}+1, \ldots, y_{B}^{0}+e_{B}\right\}, \\
& C_{B}=C_{B}^{1} \cap C_{B}^{2}, \\
& C_{L}^{1}=\left\{x_{L}^{\prime}: I_{v}\left(x_{L}^{\prime}, y_{L}^{0}\right)=0, I_{v}\left(x_{L}^{\prime}-1, y_{L}^{0}\right)=255\right\}, \\
& C_{L}^{2}=\left\{x_{L}^{0}, x_{L}^{0}-1, \ldots, x_{L}^{0}-e_{L}\right\}, \\
& C_{L}=C_{L}^{1} \cap C_{L}^{2}, \\
& C_{R}^{1}=\left\{x_{R}^{\prime}: I_{v}\left(x_{R}^{\prime}, y_{R}^{0}\right)=0, I_{v}\left(x_{R}^{\prime}+1, y_{R}^{0}\right)=255\right\}, \\
& C_{R}^{2}=\left\{x_{R}^{0}, x_{R}^{0}+1, \ldots, x_{R}^{0}+e_{L}\right\}, \\
& C_{R}=C_{R}^{1} \cap C_{R}^{2} .
\end{aligned}
$$

where $I_{v}$ is the vertical-rank filtered image in Figure. $9, e_{B}=e_{L}=e_{R}=7.000$ are the maximum differences between $y_{B}^{0}$ and $y_{b}, x_{L}^{0}$ and $x_{L}$, and $x_{R}^{0}$ and $x_{R}$, respectively. Then $y_{B}, x_{L}$, and $x_{R}$ are recalculated by (23). This research adjust the pupil centroid and radius using (24). Also, Figure. 10 shows my result in pupil recalculation. Those are mentioned as follows. 


$$
\begin{aligned}
& y_{B}=\min \left\{C_{B}\right\}, \\
& x_{L}=\max \left\{C_{L}\right\}, \\
& x_{R}=\min \left\{C_{R}\right\} . \\
& x_{0}=x_{L}+\frac{x_{R}+x_{L}}{2}, \\
& y_{0}=y_{T}+\frac{y_{B}-y_{T}}{2}, \\
& r=\frac{1}{2} \max \left\{x_{R}-x_{L}, y_{B}-y_{T}\right\} .
\end{aligned}
$$

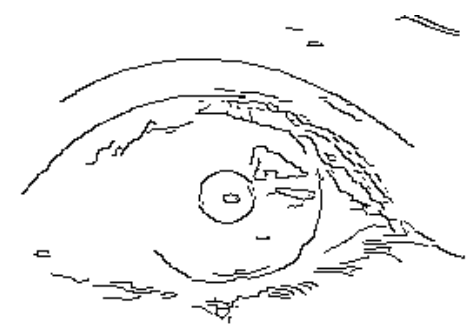

Figure 8. H-Rank filtered eye image

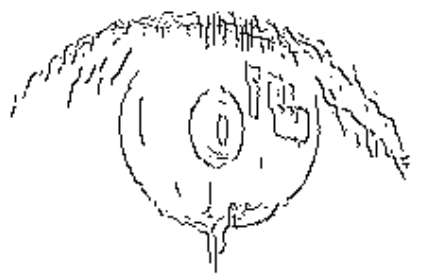

Figure 9. V-Rank filtered eye image.

\section{5). Eyelids Segmentation}

In this section, this research introduce a new method for eyelids segmentation based on Euclidean distance. Given an eye image after applying rank filter with $M=19, k=2$ and Canny algorithm with low threshold $t_{l}=50$ and high threshold $t_{h}=60$, this research eliminate all the pupil pixels using $x_{0}, y_{0}$, and $r$ in the Pupil Recalculation section as shown in Figure. 11. Instead of calculating new two means of the data points, my technique uses two fixed centroids $P_{t}\left(X, y_{t}\right)$ and $P_{b}\left(X, y_{b}\right)$ in Upper and Lower Eyelid Limits Estimation section.

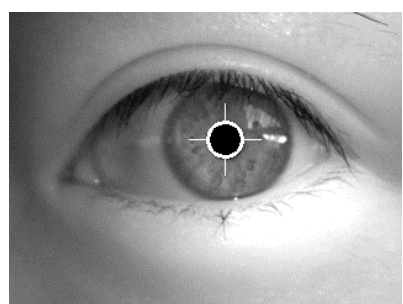

Figure 10. Pupil location. 


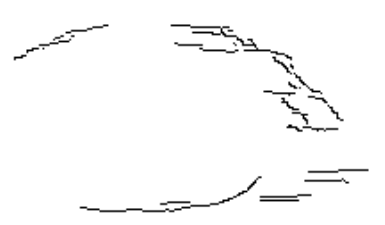

Figure 11. Eyelids segmentation.

Let $E=0.867$ be the affected parameter of $P_{t}$ and $P_{b}$ toward the eyelids data points (Figure. 12). Suppose $U$ and $L$ are the upper and lower eyelids clusters, this research partition each eyelid data point $x$ using (25). Figure. 13 shows our eyelids segmentation result.

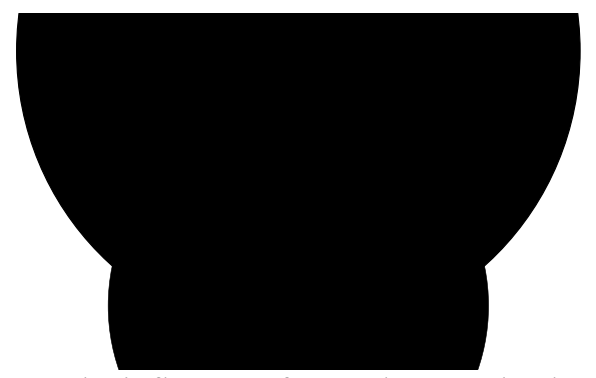

Figure 12. The influence of $P_{t}$ and $P_{b}$ on the data points.

$$
x \in \begin{cases}U & \text { if } E\left\|x-P_{t}\right\|<\left\|x-P_{b}\right\| \\ L & \text { otherwise }\end{cases}
$$

\section{6). Eyelids Parabola Fitting}

With $P_{t}, P_{b}$, and two datasets $U$ and $L$, this research find parameters for the parabola model by (26) to modelize eyelids in an eye image.

$$
y=\frac{1}{4 p}(x-h)^{2}+k .
$$

where $p$ and $(h, k)$ are focal length and vertex location (Figure. 14). In case of upper eyelid parabola modelization, based on my experiments (27). This research define $\grave{o}=10$ and $\grave{o}^{\prime}=5$ as the parameters to control the region of interest in looking for $h$ and $k$. In order to evaluate the selected parameters $q, h$, and $k$, this research calculate the sum of the squared error (28) between the eyelid actual data points and the respective ones which are measured by parabola equation with particular $q, h$, and $k$.

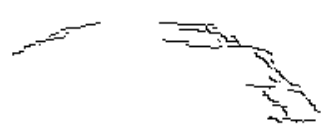

a. Upper eyelid.

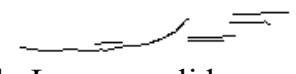

b. Lower eyelid.

Figure 13. Eyelids location. 


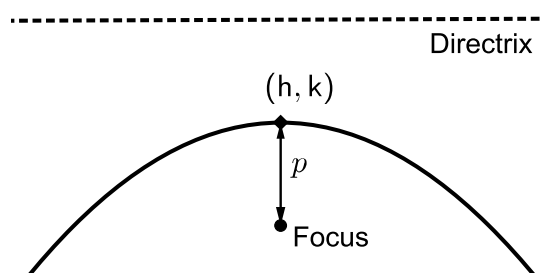

Figure 14. The parabola.

$$
\begin{gathered}
q=\frac{1}{4 p} \in\{0.0055,0.0060,0.0065,0.0070\}, \\
h \in\left\{h_{0} \in Z: X-\grave{o} \leq h_{0} \leq X+\grave{o}\right\}, \\
k \in\left\{k_{0} \in Z: y_{t}-\grave{o}^{\prime} \leq k_{0} \leq y_{t}+\grave{o}^{\prime}\right\} . \\
\operatorname{SSE}(q, h, k, U)=\sum_{i=1}^{|U|}\left(y_{i}^{\prime}-y_{i}\right)^{2} .
\end{gathered}
$$

where $|U|$ represents the number of elements in $U, y_{i}^{\prime}=q\left(x_{i}-h\right)^{2}+k$ given specific $q, h$, and $k$; and $\left(y_{i}, x_{i}\right)$ is one of the data points in $U$. Thus (28) measures the sum of the squares of differences between the estimated value $y_{i}^{\prime}$ and the actual value $y_{i}$ with respect to $x_{i}$. Then the most optimal $q, h$, and $k$ are selected by (29). Regarding lower eyelid model is (30).

$$
\begin{aligned}
& (q, h, k)=\underset{(q, h, k)}{\operatorname{argmin}} \operatorname{SSE}(q, h, k, U) . \\
& q^{\prime}=\frac{1}{4 p^{\prime}} \in\{-0.002,-0.0025\} .
\end{aligned}
$$

It is the focal length candidates. The differences between $q$ in (27) and $q^{\prime}$ in (30) indicates the effect of $P_{t}$ and $P_{b}$ on the eyelid data points which was mentioned in Eyelids Segmentation section.

By a similar way, to define candidate sets of $h^{\prime}$ and $k^{\prime}$, this research use $\dot{o}=10$ for the former and $\dot{O}^{\prime}=5$ for the latter (31), then SSE is (32).

$$
\begin{aligned}
& h^{\prime} \in\left\{h_{0} \in Z: X-\grave{o} \leq h_{0} \leq X+\grave{o}\right\}, \\
& k^{\prime} \in\left\{k_{0} \in Z: y_{b}-\grave{o}^{\prime} \leq k_{0} \leq y_{b}+\grave{o}^{\prime}\right\} . \\
& \operatorname{SSE}\left(q^{\prime}, h^{\prime}, k^{\prime}, L\right)=\sum_{i=1}^{|L|}\left(y_{i}^{\prime}-y_{i}\right)^{2} .
\end{aligned}
$$

where $y_{i}^{\prime}=q^{\prime}\left(x_{i}-h^{\prime}\right)^{2}+k^{\prime}$. Then this research use (33) to choose the parameters $q^{\prime}, h^{\prime}$, and $k^{\prime}$ for the lower parabola. Figure. 15 is our eyelids modelization result.

$$
\left(q^{\prime}, h^{\prime}, k^{\prime}\right)=\underset{\left(q^{\prime}, h^{\prime}, k^{\prime}\right)}{\operatorname{argmin}} \operatorname{SSE}\left(q^{\prime}, h^{\prime}, k^{\prime}, L\right) \text {. }
$$

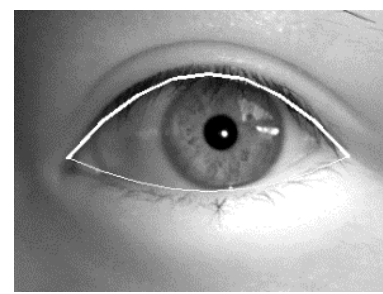

Figure. 15. Eyelids modelization 


\section{Iris Liveness Detection}

Usually, this research blink about 15-20 times [16] and make greater than or equal to 40 saccades [17] per minute. In my study, this research use two features to indicate eye movements: (1) the width/height ratio of the eye outline and (2) the distance from the pupil center to the eye centroid. Based on experiments, this research collect 50 valid eye images for classification, which ensures eye movement(s) will take place during the sampling process.

\section{1). The Width/Height Ratio of The Eye Outline}

Given $q, h, k, q^{\prime}, h^{\prime}$, and $k^{\prime}$ calculated in Eyelids Parabola Fitting section, this research estimate the width/height ratio of the eye outline $w / h$ for each valid eye image as shown in Figure. 16. Thus our feature subset for iris liveness detection is $v^{\prime}=\left(\frac{w_{1}}{h_{1}}, \frac{w_{2}}{h_{2}}, \ldots, \frac{w_{50}}{h_{50}}\right)$.

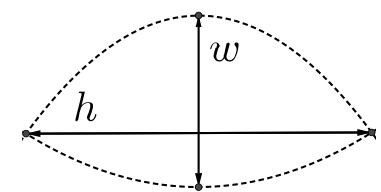

Figure. 16. The eye outline parameters.

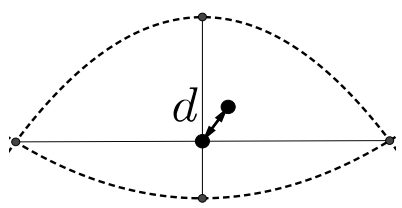

Figure. 17. Pupil location.

\section{2). The Distance from the Pupil Center to the Eye Centroid}

This research have greater than or equal to 40 saccades per minute [17]. Therefore, pupil movement is one of the most important features for iris liveness detection in my method. This research measure this attribute via the distance between the pupil center and the eye centroid (Figure. 17). Recall $v^{\prime}$ as our feature subset of eyelids variation, then the feature vector in my proposed method is $v=\left(d_{1}, d_{2}, \ldots, d_{50}, \frac{w_{1}}{h_{1}}, \frac{w_{2}}{h_{2}}, \ldots, \frac{w_{50}}{h_{50}}\right)$ where $d_{i}=\left\|p_{i}-e_{i}\right\|, \quad i=\overline{1,50}$, $p_{i}=\left(x_{0}^{i}, y_{0}^{i}\right)$, and $e_{i}=\left(X^{i}, y_{t}^{i}+\frac{y_{b}^{i}-y_{t}^{i}}{2}\right)$ are the ith pupil and eye centers, respectively.

\section{3). Iris Liveness Detection Using Gaussian Mixing Model}

Given a Gaussian Mixing Model of two Gaussian components as (34). This research use the $1^{\text {st }}$ Gaussian parameters $\theta_{1}=\left(w_{1}, \mu_{1}, \Sigma_{1}\right)$ and the 2nd ones $\theta_{2}=\left(w_{2}, \mu_{2}, \Sigma_{2}\right)$ to model liveness eye and fake eye data distributions, respectively. In order to classify a new instance $v^{\text {new }}$, this research use (35).

$$
\begin{aligned}
& f(v ; \phi)=w_{1} \mathrm{~N}\left(v ; \mu_{1}, \Sigma_{1}\right)+w_{2} \mathrm{~N}\left(v ; \mu_{2}, \Sigma_{2}\right) . \\
& \text { class }_{v^{\text {new }}}=\underset{c \in\{1,2\}}{\operatorname{argmax}}\left\{\mathrm{L}\left(v^{\text {new }}, \theta_{c}\right)\right\} .
\end{aligned}
$$

where $\mathrm{L}\left(v^{\text {new }}, \theta_{c}\right)$ is the likelihood of a set of parameters $\theta_{c}$ given an instance $v^{\text {new }}$. 


\section{4). Bagging}

Bagging is a simple ensemble method for classification improvement by combining unstable classifiers [23]. This technique works well for small datasets by repeatedly randomly resampling the training data to generate bootstrap samples. Each bootstrap sample will on average contain $63.2 \%$ of the original training set, and the rest are replicates [24]. Note that this research divide our data into training set and testing set in the ratio 7:3.

\section{Experiment and Results}

\section{A. Database}

This research store class labels, pupil movement and eyelids variation data of 244 samples using LIBSVM format [25], table 1. The number of liveness eye instances is 114 and the rest is fake eye ones. To generate a fake eye dataset, this research develop a program that uses a liveness eye digital image without post-processing to create others images using affine transformations including translation, scale, and rotation randomly as shown in Figure. 18. And, IriTech, Inc. provided us 10 fake eye image sets for testing purpose only (Figure 19).

Table 1. LIBSVM Format

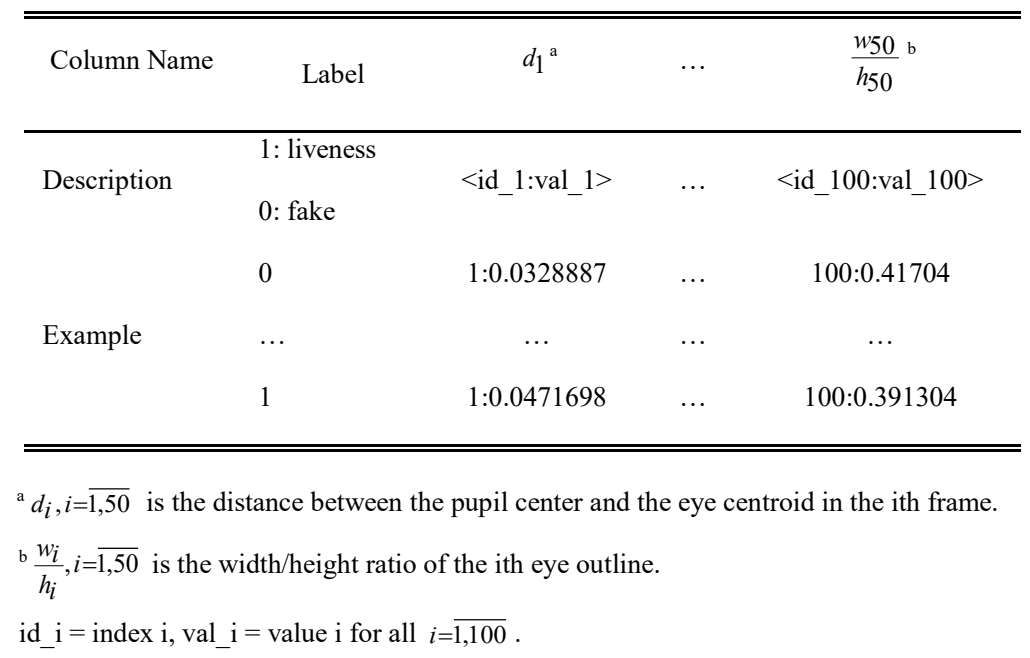

\section{B. Experiment and Results}

Let $D$ be our database. Then this research divide it into training set $T^{r}$ and testing set $T^{e}$ in the ratio 7:3 randomly. Using bootstrap aggregation, this research generate 14,000 bootstrap samples $T^{r, i}, i=\overline{1,14000}$ from $T^{r}$ to train 14,000 GMMs, respectively. After evaluating all of these GMMs using $T^{e}$, this research select 18 GMMs whose the accuracy are greater than or equal to 0.800 . Then this research pick the best combination of seven GMMs as shown in figure. 20. Although the accuracy of the best combination of five GMMs and seven GMMs are the same, according to [23], there are more unstable classifiers required for such classification problem.

On one core of an Intel(R) Core(TM) i5-2520M processor, our Windows-based C++/CLthis research application (Figure. 21) with OpenCV 2.4.6 [26] takes approximately 7.991 seconds to finish executing. This process includes eye tracking, eye features extraction and iris liveness detection. Since eye's movement based approach requires more images than the others do, the running time is longer. However, this approach works successfully with not only printed images but also static eye digital images. Moreover, the extracted features from my method can be used for further processing in an iris-based security system such as iris recognition. Figure. 22 shows my classification result with the seven selected GMMs. 

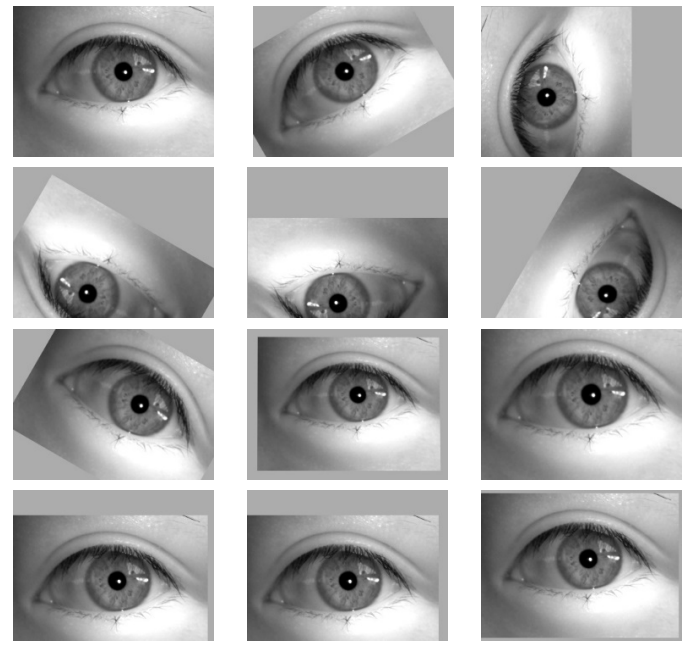

Figure 18. Fake eye image generations.

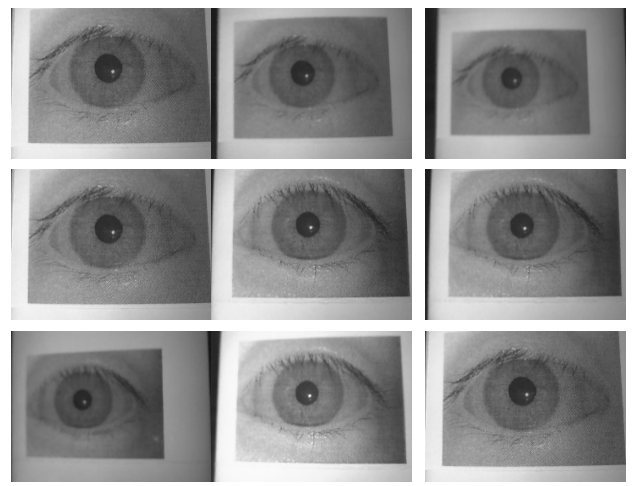

Figure 19. IriTech, Inc.'s fake eye images.

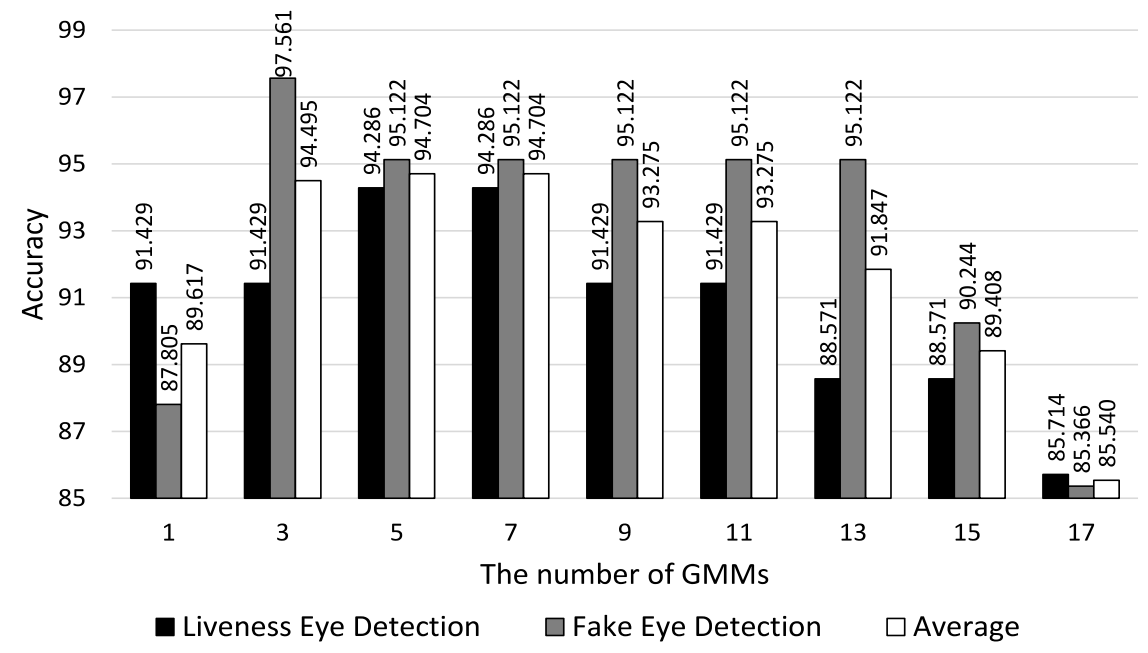

Figure 20. The accuracy of our proposed method with respect to the GMMs combination. 
Because our fake eye dataset is composed of liveness eye digital images without postprocessing, the false negative rate is 4.878 . Conversely, evaluating on the real world fake eye images by the IriTech, Inc., which consists of high quality eye printed images, the result indicates that my method is a promising one for iris liveness detection based on eye movements with false negative rate is 0.000 .

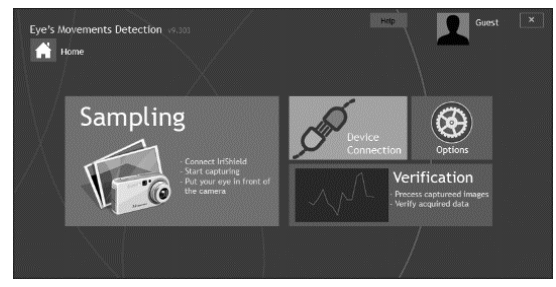

a. Application dashboard.

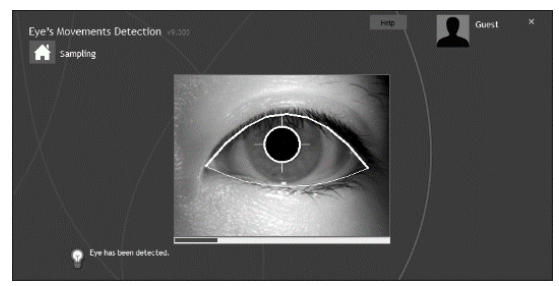

b. Sampling window.

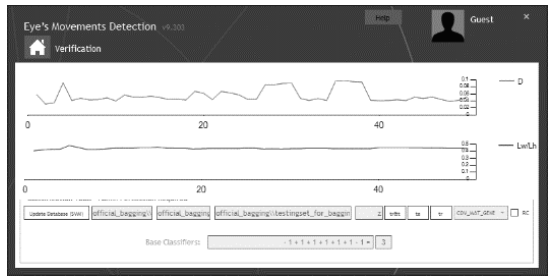

c. Verification interface with eye movement distributions.

Figure 21. Iris liveness detection application.

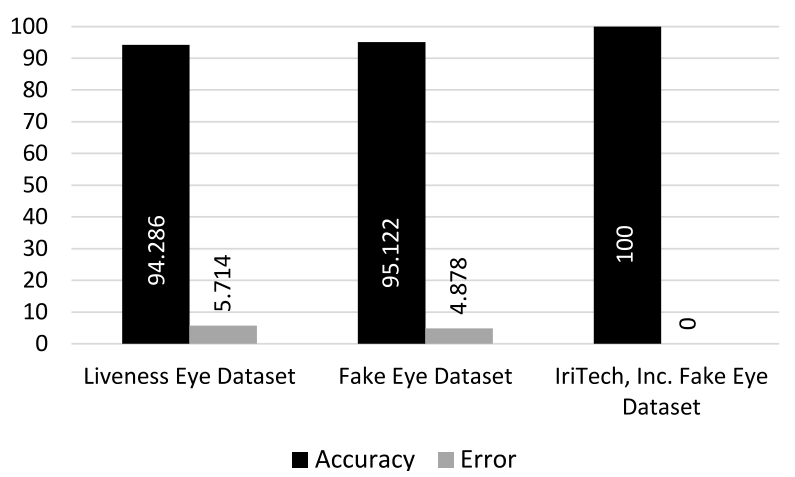

Figure 22. Our proposed method accuracy. 


\section{Conclusion}

In this paper, this research introduced a new approach for iris liveness detection based on eye movement's features. In fact, my study includes pre-processing methods for an iris-based security system such as eye detection, eye feature extraction, and iris liveness detection for both low and high quality images. The average accuracy of my proposed method on our and IriTech, Inc.'s databases is $94.704 \%$ and $100 \%$, respectively. This result indicated that my method is a novel and promising one for iris liveness detection based on eye movements with false negative rate is 0.000 on a real world database. Additionally, based on the data in eye features extraction, this research select the most informative iris image for further processing, e.g., iris recognition, etc. Our future works consist of minimizing sampling duration and taking attacks using contact lenses into account.

\section{References}

[1]. Christer Bergman, "Match-on-Card for Secure and Scalable Biometric Authentication," in Advances in Biometrics: Sensors, Algorithms and Systems of Springer, 2008, pp. 407-421.

[2]. Chandrakant D. Patel, Sanket Trivedi, and Sanjay Patel, "Biometrics in IRIS Technology: A Survey," International Journal of Scientific and Research Publications, vol. 2, pp. 1-5, 2012.

[3]. Bodade, R., and Talbar, S., "Fake iris detection: A holistic approach," International Journal of Computer Applications, vol. 19, no. 2, pp. 1-7, 2011.

[4]. Virginia Ruiz-Albacete, Pedro Tome-Gonzalez, Fernando Alonso-Fernandez, Javier Galbally, Julian Fierrez, and Javier Ortega-Garcia, "Direct Attacks Using Fake Images in Iris Verification,” Biometrics and Identity Management, vol. 5372, pp. 181-190, 2008.

[5]. Javier Galbally, Sébastien Marcel, and Julian Fierrez, "Image Quality Assessment for Fake Biometric Detection: Application to Iris, Fingerprint, and Face Recognition," IEEE Trans. Image Processing, vol. 23, no. 2, pp. 710-724, 2014.

[6]. Eui Chul Lee, Kang Ryoung Park, and Jaihie Kim, "Fake Iris Detection by Using Purkinje Image," Advances in Biometrics, vol. 3832, pp. 397-403, 2006.

[7]. Eui Chul Lee, You Jin Ko, and Kang Ryoung Park, "Fake iris detection method using Purkinje images based on gaze position," Optical Engineering, vol. 47, no. 6, pp. 067204067204, 2008.

[8]. Jonathan Connell, Nalini Ratha, James Gentile, and Ruud Bolle, "Fake iris detection using structured light," 2013 IEEE International Conference on Acoustics, Speech and Signal Processing, pp. 8692-8696, 2013, 2011.

[9]. Eui Chul Lee and Kang Ryoung Park, "Fake iris detection based on 3D structure of iris pattern," International Journal of Imaging Systems and Technology, vol. 20, no. 2, pp. $162-$ $166,2010$.

[10]. Kang Ryoung Park, Min Cheol Whang, Joa Sang Lim, and Yongjoo Cho, "Fake Iris Detection Based on Multiple Wavelet Filters and Hierarchical SVM," Information Security and Cryptology - ICISC 2006, vol. 4296, pp. 246-256, 2006.

[11]. Kang Ryoung Park, "Robust Fake Iris Detection," Articulated Motion and Deformable Objects, vol. 4069, pp. 10-18, 2006.

[12]. Rajesh Bodade and Sanjay Talbar, "Dynamic iris localisation: A novel approach suitable for fake iris detection," 2009 International Conference on Ultra Modern Telecommunications \& Workshops, pp. 1-5, 2009.

[13]. Xinyu Huang, Changpeng Ti, Qi-zhen Hou, Alade Tokuta and Ruigang Yang, "An experimental study of pupil constriction for liveness detection," 2013 IEEE Workshop on Applications of Computer Vision (WACV), pp. 252-258, 2013.

[14]. Adam Czajka, "Pupil Dynamics for Iris Liveness Detection," IEEE Trans. Information Forensics and Security, vol. 10, no. 4, pp. 726-735, 2015.

[15]. Rui Chen, Xirong Lin and Tianhuai Ding, "Liveness detection for iris recognition using multispectral images," Pattern Recognition Letters, vol. 33, no. 12, pp. 1513-1519, 2012. 
[16]. Schiffman, H.R., Sensation and Perception. An Integrated Approach (5th Edition). John Wiley \& Sons, 2001.

[17]. R. John Leigh and David S. Zee, The Neurology of Eye Movements (4 Edition). The Neurology of Eye Movements, 2006.

[18]. Hai Duong, Ung Quang Huy, Pham The Bao, and Jin Young Kim, "A Method for Selecting the Most Informative Iris Image from Real Time Video Stream," Journal of KIIT, vol. 12, pp. 61-67, 2014.

[19]. Rafael C. Gonzalez and Richard E. Woods, Digital Image Processing (2nd Edition). Prentice Hall, 2002, pp. 701-704.

[20]. Georg Heygster, "Rank filters in digital image processing," Computer Graphics and Image Processing, vol. 19, no. 2, p. 148-164, 1982.

[21]. John Canny, "A Computational Approach to Edge Detection," IEEE Trans. Pattern Analysis and Machine Intelligence, Vols. PAMI-8, no. 6, pp. 679-698, 1986.

[22]. Hiromi Kobayashi and Shiro Kohshima, "Unique morphology of the human eye and its adaptive meaning: comparative studies on external morphology of the primate eye," Journal of Human Evolution, vol. 31, no. 9, pp. 419-435, 2001.

[23]. Leo Breiman, "Bagging Predictors," Machine Learning, vol. 24, no. 2, pp. 123-140, 1996.

[24]. Thomas G. Dietterich, "Ensemble Methods in Machine Learning," Lecture Notes in Computer Science, vol. 1857, pp. 1-15, 2000.

[25]. Chih-Chung Chang and Chih-Jen Lin. LIBSVM -- A Library for Support Vector Machines. National Taiwan University. [Online]. Available: https://www.csie.ntu.edu.tw/ r94100/libsvm-2.8/README. [Accessed 25 May 2015].

[26]. Bradski G., "The OpenCV Library," Dr. Dobb's Journal of Software Tools, vol. 25, no. 11, pp. 120-126, 2000.

[27]. Javier Galbally, Jaime Ortiz-Lopez, Julian Fierrez and Javier Ortega-Garcia, "Iris Liveness Detection Based on Quality Related Features", 2012 5th IAPR International Conference on Biometrics (ICB), 2012.

[28]. Adam Czajka, Kevin W. Bowyer, "Presentation Attack Detection for Iris Recognition: An Assessment of the State of the Art", ACM Computing Surveys, Vol. 51, No. 4, 2018.

[29]. Rohit Agarwal, Anand Singh Jalal, K. V. Arya, "Enhanced Binary Hexagonal Extrema Pattern (EBHXEP) Descriptor for Iris Liveness Detection", Wireless Personal Communications, 2020.

[30]. Niladri B. Puhan, Sudha Natarajan, and A. Suhas Hegde, "Iris Liveness Detection for Semitransparent Contact Lens Spoofing", First International Conference on Digital Image Processing and Pattern Recognition, DPPR 2011 Proceedings, pp. 249 - 256, 2011.

[31]. André Anjos, Murali Mohan Chakka, Sébastien Marcel, "Motion-based counter-measures to photo attacks in face recognition”, IET Biometrics, Vol. 3, Iss. 3, pp. 147-158, 2014.

[32]. Diego Gragnaniello, Carlo Sansone, Luisa Verdoliva, "Iris liveness detection for mobile devices based on local descriptors", Pattern Recognition Letters, 2015.

[33]. Yang Hu, Konstantinos Sirlantzis, Gareth Howells, "Iris liveness detection using regional features", Pattern Recognition Letters, 2015

[34]. Federico Pala, Bir Bhanu, "Iris Liveness Detection by Relative Distance Comparisons", 2017 IEEE Conference on Computer Vision and Pattern Recognition Workshops, 2017.

[35]. Kiran B. Raja, R. Raghavendra, Christoph Busch, "Video Presentation Attack Detection in Visible Spectrum Iris Recognition Using Magnified Phase Information", IEEE Transactions on Information Forensics and Security, 2015.

[36]. Gabriela Yukari Kimura, Diego Rafael Lucio, Alceu S. Britto Jr. and David Menotti, "CNN Hyperparameter Tuning Applied to Iris Liveness Detection", arXiv preprint arXiv:2003.00833, 2020.

[37]. Adam Czajka, "Iris Liveness Detection by Modeling Dynamic Pupil Features", Advances in Computer Vision and Pattern Recognition, Handbook of Iris Recognition, 2016 
[38]. Ana F.Sequeira, JulianoMurari and JaimeS.Cardoso, "Iris Liveness Detection Methods in Mobile Applications", Proceedings of the 9th International Conference on Computer Vision Theory and Applications, pp. 22-33, 2014.

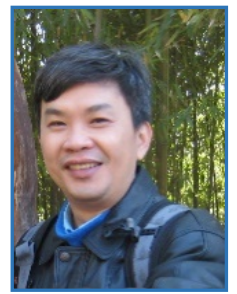

Pham The Bao received his BS in 1995, the M.Sc. in 2000, and the Ph.D. degree in Computer Science from University of Science in 2009, Vietnam. 1995 2018 Lecturer and Professor in Department of Computer Science, Faculty of Mathematics \& Computer Science, University of Science, Vietnam. 2019 now Professor and Dean of Information Science Faculty, Sai Gon University. Chair of IC-IP Lab. Research Interests: Image Processing, Pattern Recognition, Intelligent Computing. 